\title{
LIMITING WEAK TYPE ESTIMATE FOR CAPACITARY MAXIMAL FUNCTION
}

\author{
JIE XIAO AND NING ZHANG
}

\begin{abstract}
Aвstract. A capacitary analogue of the limiting weak type estimate of P. Janakiraman for the Hardy-Littlewood maximal function of an $L^{1}\left(\mathbb{R}^{n}\right)$ function (cf. [3, 4]) is discovered.
\end{abstract}

\section{Statement of Theorem}

For an $L_{l o c}^{1}$-integrable function $f$ on $\mathbb{R}^{n}, n \geq 1$, let $M f(x)$ denote the Hardy-Littlewood maximal function of $f$ at $x \in \mathbb{R}^{n}$ :

$$
M f(x)=\sup _{x \in B} \frac{1}{\mathscr{L}(B)} \int_{B}|f(y)| d y,
$$

where the supremum is taken over all Euclidean balls $B$ containing $x$ and $\mathscr{L}(B)$ stands for the $n$-dimensional Lebesgue measure of $B$. Among several results of [3, 4], P. Janakiraman obtained the following fundamental limit:

$$
\lim _{\lambda \rightarrow 0} \lambda \mathscr{L}\left(\left\{x \in \mathbb{R}^{n}: M f(x)>\lambda\right\}\right)=\|f\|_{1}=\int_{\mathbb{R}^{n}}|f(y)| d y \quad \forall \quad f \in L^{1}\left(\mathbb{R}^{n}\right) .
$$

This note studies the limiting weak type estimate for a capacity. To be more precise, recall that a set function $C(\cdot)$ on $\mathbb{R}^{n}$ is said to be a capacity (cf. [1, 2]) provided that

$$
\left\{\begin{array}{l}
C(\emptyset)=0 ; \\
0 \leq C(A) \leq \infty \quad \forall \quad A \subseteq \mathbb{R}^{n} \\
C(A) \leq C(B) \quad \forall \quad A \subseteq B \subseteq \mathbb{R}^{n} ; \\
C\left(\cup_{i=1}^{\infty} A_{i}\right) \leq \sum_{i=1}^{\infty} C\left(A_{i}\right) \quad \forall \quad A_{i} \subseteq \mathbb{R}^{n}
\end{array}\right.
$$

For a given capacity $C(\cdot)$ let

$$
M_{C} f(x)=\sup _{x \in B} \frac{1}{C(B)} \int_{B}|f(y)| d y
$$

2000 Mathematics Subject Classification. Primary 42B25; Secondary 46B70, 28A12. Key words and phrases. Limiting weak type estimate; Capacitary maximal function. Project supported by NSERC of Canada as well as by URP of Memorial University, Canada. 
be the capacitary maximal function of an $L_{l o c}^{1}$-integrable function $f$ at $x$ for which the supremum ranges over all Euclidean balls $B$ containing $x$; see also [5].

In order to establish a capacitary analogue of the last limit formula for $f \in L^{1}\left(\mathbb{R}^{n}\right)$, we are required to make the following natural assumptions:

- Assumption 1 - the capacity $C(B(x, r))$ of the ball $B(x, r)$ centered at $x$ with radius $r$ is a function depending on $r$ only, but also the capacity $C(\{x\})$ of the set $\{x\}$ of a single point $x \in \mathbb{R}^{n}$ equals 0 .

- Assumption 2 - there are two nonnegative functions $\phi$ and $\psi$ on $(0, \infty)$ such that

$\left\{\begin{array}{l}\phi(t) C(E) \leq C(t E) \leq \psi(t) C(E) \quad \forall t>0 \& t E=\left\{t x \in \mathbb{R}^{n}: x \in E \subseteq \mathbb{R}^{n}\right\} ; \\ \lim _{t \rightarrow 0} \phi(t)=0=\lim _{t \rightarrow 0} \psi(t) \& \lim _{t \rightarrow 0} \psi(t) / \phi(t)=\tau \in(0, \infty) .\end{array}\right.$

Here, it is worth mentioning that the so-called $p$-capacity satisfies all the assumptions; see also [6].

Theorem 1.1. Under the above-mentioned two assumptions, one has

$$
\lim _{\lambda \rightarrow 0} \lambda C\left(\left\{x \in \mathbb{R}^{n}: M_{C} f(x)>\lambda\right\}\right) \approx\|f\|_{1} \quad \forall \quad f \in L^{1}\left(\mathbb{R}^{n}\right) .
$$

Here and henceforth, $\mathrm{X} \approx \mathrm{Y}$ means that there is a constant $c>0$ independent of $\mathrm{X}$ and $\mathrm{Y}$ such that $c^{-1} \mathrm{Y} \leq \mathrm{X} \leq c \mathrm{Y}$.

\section{Four Lemmas}

To prove Theorem 1.1, we will always suppose that $C(\cdot)$ is a capacity obeying Assumptions 1-2 above, but also need four lemmas based on the following capacitary maximal function $M_{C} v$ of a finite nonnegative Borel measure $v$ on $\mathbb{R}^{n}$ :

$$
M_{C} v(x)=\sup _{B \ni x} \frac{v(B)}{C(B)} \quad \forall \quad x \in \mathbb{R}^{n},
$$

where the supremum is taken over all balls $B \subseteq \mathbb{R}^{n}$ containing $x$.

Lemma 2.1. If $\delta_{0}$ is the delta measure at the origin, then

$$
C\left(\left\{x \in \mathbb{R}^{n}: M_{C} \delta_{0}(x)>\lambda\right\}\right)=\frac{1}{\lambda} .
$$

Proof. According to the defintion of the delta measure and Assumptions 1-2, we have

$$
M_{C} \delta_{0}(x)=\frac{1}{C(B(x,|x|))} \quad \forall \quad|x| \neq 0 .
$$

Now, if $x$ obeys $M_{C} \delta_{0}(x)>\lambda$, then

$$
C(B(x,|x|))<\frac{1}{\lambda}
$$


Note that if $C(B(0, r))$ equals $\frac{1}{\lambda}$, then one has the following property:

$$
\left\{\begin{array}{lll}
C(B(x,|x|))<\frac{1}{\lambda} & \forall & |x|<r \\
C(B(x,|x|))=\frac{1}{\lambda} & \forall & |x|=r \\
C(B(x,|x|))>\frac{1}{\lambda} & \forall & |x|>r
\end{array}\right.
$$

Therefore,

$$
\left\{x \in \mathbb{R}^{n}: M_{C} \delta_{0}(x)>\lambda\right\}=B(0, r),
$$

and consequently,

$$
C\left(\left\{x \in \mathbb{R}^{n}: M_{C} \delta_{0}(x)>\lambda\right\}\right)=C(B(0, r))=\frac{1}{\lambda} .
$$

Lemma 2.2. If $v$ is a finte nonnegative Borel measure on $\mathbb{R}^{n}$ with $v\left(\mathbb{R}^{n}\right)=1$, then

$$
\lim _{t \rightarrow 0} C\left(\left\{x \in \mathbb{R}^{n}: M_{C} v_{t}(x)>\lambda\right\}\right)=\frac{1}{\lambda},
$$

where

$$
\left\{\begin{array}{l}
t>0 \\
v_{t}(E)=v\left(\frac{1}{t} E\right) \\
\frac{1}{t} E=\left\{\frac{x}{t}: x \in E\right\} \\
E \subseteq \mathbb{R}^{n}
\end{array}\right.
$$

Proof. For two positive numbers $\epsilon$ and $\eta$, choose $\epsilon_{1}$ small relative to both $\epsilon$ and $\eta$, but also let $t$ be small and the induced $\epsilon_{t}$ be such that

$$
\left\{\begin{array}{l}
v_{t}\left(B\left(0, \epsilon_{t}\right)\right)>1-\epsilon ; \\
\epsilon_{t}=3^{-1} \epsilon_{1} ; \\
\lim _{t \rightarrow 0} \epsilon_{t}=0 ; \\
\epsilon<\eta C\left(B\left(0, \epsilon_{1}\right)\right) .
\end{array}\right.
$$

Now, if

$$
\left\{\begin{array}{l}
E_{1, \lambda}^{t}=\left\{x \in \mathbb{R}^{n} \backslash B\left(0, \epsilon_{1}\right): \lambda<M_{C} v_{t}(x) \leq \frac{1}{C\left(B\left(x,|x|-\epsilon_{t}\right)\right.}\right\} \\
E_{2, \lambda}^{t}=\left\{x \in \mathbb{R}^{n} \backslash B\left(0, \epsilon_{1}\right): \max \left\{\lambda, \frac{1}{C\left(B\left(x,|x|-\epsilon_{t}\right)\right.}\right\}<M_{C} v_{t}(x)\right\},
\end{array}\right.
$$

then

$$
E_{1, \lambda}^{t} \cup E_{2, \lambda}^{t} \cup B\left(0, \epsilon_{1}\right)=\left\{x \in \mathbb{R}^{n}: M_{C} v_{t}(x)>\lambda\right\} .
$$

On the one hand, for such $x \in E_{2, \lambda}^{t}$ and $\forall \tilde{r}>0$ that

$$
\frac{v_{t}(B(x, \tilde{r}))}{C\left(B\left(x,|x|-\epsilon_{t}\right)\right)} \leq \frac{1}{C\left(B\left(x,|x|-\epsilon_{t}\right)\right)}<M_{C} v_{t}(x) .
$$


Additionally, since for any $r_{1}, r_{2}$ satisfying $0 \leq r_{1} \leq r_{2}$,

$$
C\left(B\left(x, r_{1}\right)\right) \leq C\left(B\left(x, r_{2}\right)\right),
$$

$C(B(x, r))$ is an increasing function with respect to $r$. There exists $r<|x|-\epsilon_{t}$ such that

$$
\frac{v_{t}(B(x, r))}{C\left(B\left(x,|x|-\epsilon_{t}\right)\right)} \leq \frac{v_{t}(B(x, r))}{C(B(x, r))} \leq M_{C} v_{t}(x),
$$

and hence by the Assumption 1, for any $x_{i} \in E_{2, \lambda}^{t}$ there exists $r_{i}>0$ such that

$$
r_{i}<\left|x_{i}\right|-\epsilon_{t} \& \lambda \leq \frac{v_{t}\left(B\left(x_{i}, r_{i}\right)\right)}{C(B(x, r))} .
$$

By the Wiener covering lemma, there exists a disjoint collection of such balls $B_{i}=B\left(x_{i}, r_{i}\right)$ and a constant $\alpha>0$ such that

$$
\cup_{i} B_{i} \subseteq E_{2, \lambda}^{t} \subseteq \cup_{i} \alpha B_{i},
$$

Therefore, we get a constant $\gamma>0$, which only depends on $\alpha$, such that

$$
C\left(E_{2, \lambda}^{t}\right) \leq \gamma \sum_{i} C\left(B_{i}\right)<\gamma \sum_{i} \frac{v_{t}\left(B_{i}\right)}{\lambda} \leq \frac{\gamma \epsilon}{\lambda},
$$

thanks to

$$
B_{i} \cap B\left(0, \epsilon_{t}\right)=\emptyset \& 1-v_{t}\left(B\left(0, \epsilon_{t}\right)\right)<\epsilon .
$$

On the other hand, if $x \in E_{1, \lambda}^{t}$, then

$$
\begin{aligned}
\frac{1-\epsilon}{C\left(B\left(x,|x|+\epsilon_{t}\right)\right)} & \leq \frac{v_{t}\left(B\left(x,|x|+\epsilon_{t}\right)\right)}{C\left(B\left(x,|x|+\epsilon_{t}\right)\right)} \\
& \leq M_{C} v_{t}(x) \\
& \leq \frac{1}{C\left(B\left(x,|x|-\epsilon_{t}\right)\right)}
\end{aligned}
$$

Since

for $\eta>0$ there exists $T>0$ such that

$$
\left\{\begin{array}{l}
\lim _{t \rightarrow 0}\left(\frac{1}{C\left(B\left(x,|x|+\epsilon_{t}\right)\right.}-\frac{1}{C\left(B\left(x,|x|-\epsilon_{t}\right)\right.}\right)=0 \\
\lim _{t \rightarrow 0}\left(\frac{1}{C\left(B\left(x,|x|+\epsilon_{t}\right)\right.}-\frac{1}{C(B(x,|x|))}\right)=0,
\end{array}\right.
$$

$$
\begin{aligned}
\left|M_{C} v_{t}(t)-M_{C} \delta_{0}\right| & <\eta+\frac{\epsilon}{C(B(0,|x|))} \\
& <\eta+\frac{\epsilon}{C\left(B\left(0, \epsilon_{1}\right)\right)} \\
& <2 \eta \quad \forall t \in(0, T) .
\end{aligned}
$$

Note that

$$
M_{C} \delta_{0}(x)-2 \eta \leq M_{C} v_{t} \leq M_{C} \delta_{0}(x)+2 \eta \quad \forall \quad x \in E_{1, \lambda}^{t} .
$$


Thus

$$
\left\{x \in \mathbb{R}^{n}: M_{C} \delta_{0}(x)>\lambda+2 \eta\right\} \subseteq E_{1, \lambda}^{t} \subseteq\left\{x \in \mathbb{R}^{n}: M_{C} \delta_{0}(x)>\lambda+2 \eta\right\} .
$$

This in turn implies

$$
\begin{aligned}
& C\left(\left\{x \in \mathbb{R}^{n}: M_{C} \delta_{0}(x)>\lambda+2 \eta\right\}\right) \\
& \quad \leq C\left(E_{1, \lambda}^{t}\right) \\
& \quad \leq C\left(\left\{x \in \mathbb{R}^{n}: M_{C} \delta_{0}(x)>\lambda+2 \eta\right\}\right) .
\end{aligned}
$$

Now, an application of Lemma 2.1 yields

$$
\frac{1}{\lambda+2 \eta} \leq C\left(\left\{x \in \mathbb{R}^{n}: M_{C} v_{t}(x)>\lambda\right\} \cap\left(\mathbb{R}^{n} \backslash B\left(0, \epsilon_{1}\right)\right)\right) \leq \frac{1}{\lambda-2 \eta}+\frac{\gamma \epsilon}{\lambda} .
$$

Letting $t \rightarrow 0$ and using Assumption 1, we get

$$
\lim _{t \rightarrow 0} C\left(\left\{x \in \mathbb{R}^{n}: M_{C} v_{t}(x)>\lambda\right\}\right)=\frac{1}{\lambda} .
$$

Lemma 2.3. If $v$ is a nonnegative Borel measure on $\mathbb{R}^{n}$, then $M_{C} v(x)$ is upper semi-continuous.

Proof. According to the definition of $M_{C} v(x)$, there exists a radius $r$ corresponding to $M_{C} v(x)>\lambda>0$ such that

$$
\frac{v(B(x, r))}{C(B(x, r))}>\lambda \text {. }
$$

For a slightly larger number $s$ with $\lambda+\delta>s>r$, we have

$$
\frac{v(B(x, r))}{C(B(x, s))}>\lambda \text {. }
$$

Then applying Assumption 1, for any $z$ satisfying $|z-x|<\delta$,

$$
M_{C} v(z) \geq \frac{v(B(z, s))}{C(B(z, s))} \geq \frac{v(B(x, r))}{C(B(x, s))}>\lambda
$$

Thereby, the set $\left\{x \in \mathbb{R}^{n}: M_{C} v(x)>\lambda\right\}$ is open, as desired.

Lemma 2.4. If $v$ is a finite nonnegative Borel measure on $\mathbb{R}^{n}$, then there exists a constant $\gamma>0$ such that

$$
\lambda C\left(\left\{x \in \mathbb{R}^{n}: M_{C} v(x)>\lambda\right\}\right) \leq \gamma \nu\left(\mathbb{R}^{n}\right) .
$$

Proof. Following the argument for [7, Page 39, Theorem 5.6], we set $E_{\lambda}=$ $\left\{x \in \mathbb{R}^{n}: M_{C} v(x)>\lambda\right\}$, and then select a $v$-measurable set $E \subseteq E_{\lambda}$ with $v(E)<\infty$. Lemma 2.3 proves that $E_{\lambda}$ is open. Therefore, for each $x \in E$, there exists an $x$-related ball $B_{x}$ such that

$$
\frac{v\left(B_{x}\right)}{C\left(B_{x}\right)}>\lambda \text {. }
$$


A slight modification of the proof of [7, Page 39, Lemma 5.7] applied to the collection of balls $\left\{B_{x}\right\}_{x \in E}$, and Assumption 2, show that we can find a sub-collection of disjoint balls $\left\{B_{i}\right\}$ and a constant $\gamma>0$ such that

$$
C(E) \leq \gamma \sum_{i} C\left(B_{i}\right) \leq \sum_{i} \frac{\gamma}{\lambda} v\left(B_{i}\right) \leq \frac{\gamma}{\lambda} v\left(\mathbb{R}^{n}\right) .
$$

Note that $E$ is an arbitrary subset of $E_{\lambda}$. Thereby, we can take the supremum over all such $E$ and then get

$$
C\left(E_{\lambda}\right)<\frac{\gamma}{\lambda} \nu\left(\mathbb{R}^{n}\right)
$$

\section{Proof of Theorem}

First of all, suppose that $v$ is a finite nonnegative Borel measure on $\mathbb{R}^{n}$ with $v\left(\mathbb{R}^{n}\right)=1$. According to the definition of the capacitary maximal function, we have

$$
M_{C} v_{t}(x)=\sup _{r>0} \frac{v_{t}(B(x, r))}{C(B(x, r))}=\sup _{r>0} \frac{v\left(B\left(\frac{x}{t}, \frac{r}{t}\right)\right)}{C\left(t B\left(\frac{x}{t}, \frac{r}{t}\right)\right)} .
$$

From Assumption 2 it follows that

$$
\frac{M_{C} v\left(\frac{x}{t}\right)}{\psi(t)} \leq M_{C} v_{t}(x) \leq \frac{M_{C} v\left(\frac{x}{t}\right)}{\phi(t)},
$$

and such that

$$
\begin{aligned}
\left\{x \in \mathbb{R}^{n}: M_{C} v\left(\frac{x}{t}\right)>\lambda \psi(t)\right\} & \subseteq\left\{x \in \mathbb{R}^{n}: M_{C} v_{t}(x)>\lambda\right\} \\
& \subseteq\left\{x \in \mathbb{R}^{n}: M_{C} v\left(\frac{x}{t}\right)>\lambda \phi(t)\right\} .
\end{aligned}
$$

The last inclusions give that

$$
\begin{aligned}
& \frac{\phi(t)}{\psi(t)} \lambda \psi(t) C\left(\left\{x \in \mathbb{R}^{n}: M_{C} v(x)>\lambda \psi(t)\right\}\right) \\
& \quad \leq \lambda \phi(t) C\left(\left\{x \in \mathbb{R}^{n}: M_{C} v(x)>\lambda \psi(t)\right\}\right) \\
& \quad \leq \lambda C\left(\left\{t x \in \mathbb{R}^{n}: M_{C} v(x)>\lambda \psi(t)\right\}\right) \\
& \quad=\lambda C\left(\left\{x \in \mathbb{R}^{n}: M_{C} v(x / t)>\lambda \psi(t)\right\}\right) \\
& \quad \leq \lambda C\left(\left\{x \in \mathbb{R}^{n}: M_{C} v_{t}(x)>\lambda\right\}\right) \\
& \quad \leq \lambda C\left(\left\{x \in \mathbb{R}^{n}: M_{C} v(x / t)>\lambda \phi(t)\right\}\right) \\
& \quad=\lambda C\left(\left\{t x \in \mathbb{R}^{n}: M_{C} v(x)>\lambda \phi(t)\right\}\right) \\
& \quad \leq \lambda \psi(t) C\left(\left\{x \in \mathbb{R}^{n}: M_{C} v(x)>\lambda \phi(t)\right\}\right) \\
& \quad \leq \frac{\psi(t)}{\phi(t)} \lambda \phi(t) C\left(\left\{x \in \mathbb{R}^{n}: M_{C} v(x)>\lambda \phi(t)\right\}\right) .
\end{aligned}
$$


These estimates and Lemma 2.2, plus applying Assumption 2 and letting $t \rightarrow 0$, in turns derive

$$
\begin{aligned}
\tau^{-1} & \leq \liminf _{\lambda \rightarrow 0} \lambda C\left(\left\{x \in \mathbb{R}^{n}: M_{C} v(x)>\lambda\right\}\right) \\
& \leq \limsup _{\lambda \rightarrow 0} \lambda C\left(\left\{x \in \mathbb{R}^{n}: M_{C} v(x)>\lambda\right\}\right) \leq \tau
\end{aligned}
$$

Next, let

$$
h(\lambda)=\lambda C\left(\left\{x \in \mathbb{R}^{n}: M_{C} v>\lambda\right\}\right)
$$

By Lemma 2.4 and the last estimate for both the limit inferior and the limit superior, there exists two constants $A>0$ and $\lambda_{0}>0$ such that

$$
A \leq h(\lambda) \leq \gamma \quad \forall \quad \lambda \in\left(0, \lambda_{0}\right)
$$

Moreover, for any given $\varepsilon>0$, choose a sequence $\left\{y_{i}=\left[\frac{\gamma}{A}(1-\varepsilon)^{N}\right]^{i}\right\}_{1}^{\infty}$, where $N$ is a natural number satisfying $\frac{\gamma}{A}(1-\varepsilon)^{N}<1$. Then, there exists an integer $N_{0} \geq 1$, such that $y_{N_{0}}<\lambda_{0}$. Hence, for any $n>m>N_{0}$ we have

$$
\begin{aligned}
& \left|h\left(y_{m}\right)-h\left(y_{n}\right)\right| \\
& \quad \leq\left|y_{m} C\left(\left\{x \in \mathbb{R}^{n}: M_{C} v(x)>y_{m}\right\}\right)-y_{n} C\left(\left\{x \in \mathbb{R}^{n}: M_{C} v(x)>y_{n}\right\}\right)\right| \\
& \quad \leq\left|y_{m}-y_{n}\right| C\left(\left\{x \in \mathbb{R}^{n}: M_{C} v(x)>y_{m}\right\}\right) \\
& \quad+y_{n}\left|C\left(\left\{x \in \mathbb{R}^{n}: M_{C} v(x)>y_{m}\right\}\right)-C\left(\left\{x \in \mathbb{R}^{n}: M_{C} v(x)>y_{n}\right\}\right)\right| \\
& \quad \leq\left|y_{m}-y_{n}\right| \frac{\gamma}{y_{m}}+y_{n}\left|\frac{\gamma}{y_{n}}-\frac{A}{y_{m}}\right| \\
& \quad \leq \gamma\left(1-\frac{y_{n}}{y_{m}}\right)+\left(\gamma-A \frac{y_{n}}{y_{m}}\right) \\
& \leq \gamma\left(1-\left[\frac{\gamma}{A}(1-\varepsilon)^{N}\right]^{n-m}\right)+\left(\gamma-A\left[\frac{\gamma}{A}(1-\varepsilon)^{N}\right]^{n-m}\right) \\
& \leq \gamma\left(1-(1-\varepsilon)^{N(n-m)}\right)+\left(\gamma-\gamma(1-\varepsilon)^{N(n-m)}\right) \\
& \leq 2 \gamma N(n-m) \varepsilon .
\end{aligned}
$$

Consequently, $\left\{h\left(y_{i}\right)\right\}$ is a Cauchy sequence, $D=\lim _{i \rightarrow \infty} h\left(y_{i}\right)$ exists. Note that for any small $\lambda$ there exists a large $i$ such that

$$
y_{i+1} \leq \lambda \leq y_{i}
$$


Thereby, from the triangle inequality it follows that if $i$ is large enough then

$$
\begin{aligned}
|h(\lambda)-D| & \leq\left|h(\lambda)-h\left(y_{i}\right)\right|+\left|h\left(y_{i}\right)-D\right| \\
& \leq\left|y_{i}-\lambda\right| \frac{\gamma}{y_{i}}+\lambda\left|\frac{\gamma}{\lambda}-\frac{A}{y_{i}}\right|+\left|h\left(y_{i}\right)-D\right| \\
& \leq \gamma\left(1-\frac{\lambda}{y_{i}}\right)+\left(\gamma-A \frac{\lambda}{y_{i}}\right)+\left|h\left(y_{i}\right)-D\right| \\
& \leq \gamma\left(1-\frac{y_{i+1}}{y_{i}}\right)+\left(\gamma-A \frac{y_{i+1}}{y_{i}}\right)+\left|h\left(y_{i}\right)-D\right| \\
& \leq(2 \gamma N+1) \varepsilon
\end{aligned}
$$

This in turn implies that $\lim _{\lambda \rightarrow 0} \lambda C\left(\left\{x \in \mathbb{R}^{n}: M_{C} v(x)>\lambda\right\}\right)$ exists, and consequently,

$$
\tau^{-1} \leq \lim _{\lambda \rightarrow 0} \lambda C\left(\left\{x \in \mathbb{R}^{n}: M_{C} v(x)>\lambda\right\}\right) \leq \tau
$$

holds.

Finally, upon employing the given $L^{1}\left(\mathbb{R}^{n}\right)$ function $f$ with $\|f\|_{1}>0$ to produce a finite nonnegative measure $v$ with $v\left(\mathbb{R}^{n}\right)=1$ via

$$
v(E)=\frac{1}{\|f\|_{1}} \int_{E}|f(y)| d y \quad \forall \quad E \subseteq \mathbb{R}^{n},
$$

we obtain

$$
\lim _{\lambda \rightarrow 0} \lambda C\left(\left\{x \in \mathbb{R}^{n}: M_{C} f(x)>\lambda\|f\|_{1}\right\}\right) \approx 1,
$$

thereby getting

$$
\lim _{\lambda \rightarrow 0} \lambda\|f\|_{1} C\left(\left\{x \in \mathbb{R}^{n}: M_{C} f(x)>\lambda\|f\|_{1}\right\}\right) \approx\|f\|_{1} .
$$

By setting $\tilde{\lambda}=\lambda\|f\|_{1}$ in the last estimate, we reach the desired result.

\section{REFERENCES}

[1] D. R. Adams, Choquet integrals in potential theory. Publ. Mat. 42 (1998), 3-66.

[2] I. Asekritova, J. Cerda, N. Kruglyak, The Riesz-Herz equivalence for capacity maximal functions. Rev. Mat. Complut. 25 (2012), 43-59.

[3] P. Janakiraman, Limiting weak-type behavior for singular integral and maximal operators. Trans. Amer. Math. Soc. 358 (2006), 1937-1952.

[4] P. Janakiraman, Limiting weak-type behavior for the Riesz transform and maximal operator when $\lambda \rightarrow \infty$. Michigan Math. J. 55 (2007), 35-50.

[5] J. Kinnunen, The Hardy-Littlewood maximal function of a Sobolev function. Israel J. Math. 100 (1997), 117-224.

[6] J. Xiao, Carleson embeddings for Sobelev spaces via heat equation. J. Differential Equations 224 (2006), 277-295.

[7] R. Brown, Lecture notes: harmonic analysis. http://www.ms.uky.edu/ rbrown/courses /ma773/notes.pdf. 
Jie Xiao, Department of Mathematics and Statistics, Memorial University of NewFoundLAND, St. John's, NL A1C 5S7, CANADA

E-mail address: jxiao@mun.ca

Ning Zhang, Department of Mathematics and Statistics, Memorial University of NewFoundland, ST. John's, NL A1C 5S7, Canada

E-mail address: nz7701@mun.ca 